\title{
Caries and periodontal disease in cancer survivors
}

\section{Abstracted from}

Hong CH, Napeñas J, Hodgson BD, Stokman MA, Mathers-Stauffer V, Elting LS, Spijkervet FK, Brennan MT

Dental Disease Section, Oral Care Study Group, Multi-national Association of Supportive Care in Cancer (MASCC)/International Society of Oral Oncology (ISOO). A systematic review of dental disease in patients undergoing cancer therapy. Support Care Cancer 2010 Aug; 18: 1007-1021. Epub 2010 May 7.

Address for correspondence: Department of Oral Medicine, Carolinas Medical Center, Charlotte, NC 28232, USA. E-mail: catherine.hong@carolinashealthcare.org

\section{Question: What is the prevalence of caries, periodontal disease and local and systemic odontogenic infections in cancer survivors?}

Data sources PubMed and EMBASE were searched for articles published between 1 January 1990 and 31 December 2008. Study selection Eligible studies were those that included dental caries and periodontal disease and pre-cancer dental clearance protocols in cancer patients undergoing head and neck radiotherapy, chemotherapy or combined treatment modalities. Rather than any study inclusion criteria this review had a number of exclusion criteria instead: systematic and non-systematic reviews; microbiology studies; growth and development studies; organ transplant studies; studies eliciting dental complications through questionnaires, studies reporting data from previous publications; phase I and II studies; opinion papers and case reports; articles published before 1990. All reports also had to be in English.

Data extraction and synthesis Each article was reviewed by two authors independently with pilot-tested data collection forms. No quality assessment was pre-specified.

Results Sixty-four articles were included. Forty-six of these were observational (24 cohort, eight case control, 14 cross-sectional), and 18 were interventional studies. It is unclear how many participants this included. A weighted prevalence of caries was reported but it is unclear how the weighting was calculated. Overall the weighted prevalence was $28.1 \%$. The overall DMFT for patients who were postantineoplastic therapy was 9.19 (SD, 7.98; $\mathrm{n}=457$ ). The overall plaque index for patients who were post-antineoplastic therapy was 1.38 (SD, $0.25 ; \mathrm{n}=189$ ).

Conclusions Patients who were post-radiotherapy had the highest DMFT compared to those who were post-chemotherapy and healthy controls.

\section{Commentary}

Oral complications associated with cancer chemotherapy and radiation result from complex interactions among multiple factors. ${ }^{1,2}$ The most prominent contributors are direct damage to oral structures (soft and hard tissue), attenuation of immune and other protective systems, and interference with normal healing. Principal causes can be attributed to both direct stomatotoxicity and indirect stomatotoxicity. Direct toxicities are initiated via primary injury to oral tissues. Indirect toxicities are caused by non-oral toxicities that secondarily affect the oral cavity, including myelosuppression, loss of tissue-based immune cells, and loss of protective salivary constituents. ${ }^{3}$

These oral complications may occur during and after cancer therapy and are generally grouped into two broad categories: acute and chronic. In general, intensive chemotherapy regimens cause acute toxicities that following discontinuation of therapy, damaged tissues recover. In contrast, radiation protocols may cause not only acute oral toxicities, but induce permanent tissue damage that results in a lifelong risk for the patient. Ionising radiation to salivary glands results in inflammatory and degenerative effects on salivary gland parenchyma. Salivary flow decreases within one week of starting radiation treatment and xerostomia becomes apparent when doses exceed $10 \mathrm{~Gy}$. Doses >54 Gy are generally considered to induce irreversible dysfunction. ${ }^{4}$

This systematic review of dental disease as an oral complication of oncological treatments was aimed to evaluate the literature and update understanding of the impact of present cancer therapies on the dental apparatus and the role of pre-cancer treatment dental protocols since the 1989 NIH Development Consensus Conference on the Oral Complications of Cancer Therapies. ${ }^{5}$ Its specific goals included determining the prevalence of dental caries and the prevalence of periodontal disease in cancer survivors, and the prevalence of local and systemic infections caused by dental disease during cancer therapy.

\section{Review methodology}

EMBASE and MEDLINE databases were searched for articles published between 1 January 1990 and 31 December 2008. Primary outcome for eligible studies was to retrieve all literature containing original data on dental caries and periodontal disease and pre-cancer dental protocols in cancer patients undergoing chemotherapy, head and neck radiotherapy or combined treatment modalities. Each article was reviewed by two authors independently with pilottested data collection forms. Exclusion criteria for selected stud- 
ies were listed as follows: systematic and non-systematic reviews; microbiology studies; growth and development studies; organ transplant studies; studies eliciting dental complications through questionnaires; studies reporting data from previous publications; phase I and II studies, opinion papers and case reports; articles published before 1990 .

Of the 64 studies included, 46 were observational and 18 interventional. In regards to the age of the population sampled, 31 studies recruited adults, 24 recruited children, four included both paediatric and adult patients and age was not stated in five studies. The two most common malignancies in the review were head and neck cancer and haematological malignancies. Concerning the treatment modality listed in the 64 studies, 22 studies ( $\mathrm{n}=710 \mathrm{pts}$ ) included chemotherapy only; 30 studies included radiation only ( $\mathrm{n}=3477 \mathrm{pts}$ ); 19 studies included radiation and chemotherapy ( $\mathrm{n}=696 \mathrm{pts}$ ), whereas in seven studies ( $\mathrm{n}=1812 \mathrm{pts})$ there was no breakdown or a vague description of administered cancer therapy.

Prevalence of dental and periodontal disease in cancer survivors Of the 46 observational studies that were included to evaluate the weighted prevalence of dental disease, 24 were cohort, eight case control and 14 cross-sectional studies.

The overall weighted prevalence of dental caries was $28.1 \%$ and was determined from 19 studies. Unexpectedly, the weighted prevalence of dental caries in patients who only received chemotherapy seemed to be highest $(37.3 \%, 95 \% \mathrm{CI} 0-85.7)$ when compared to those who received radiotherapy $(24.1 \%, 95 \%$ CI $0-66.2)$ or to patients receiving chemoradiotherapy $(21.4 \%$, 95\%CI 6.9 35.8). Authors considered two reasons for this unanticipated caries prevalence. The first explanation is that patients undergoing head and neck radiotherapy are at lifelong risk of developing osteoradionecrosis, thus dental management protocols in these patients may require more aggressive approaches (ie dental extractions) than those being prepared for chemotherapy. The other reason explained by the authors is that the majority of the studies (12 of the 19 studies) were conducted in paediatric populations with a high proportion of haematologic malignancies requiring high doses of chemotherapy, and who frequently take sugar-rich medications or consume cariogenic dietary supplements. The overall DMFT for patients who were post-cancer therapy was 9.19(SD 7.98; $\mathrm{n}=457$ ). When DMFT was estimated by treatment groups, patients who were post-radiotherapy presented higher index values than those who received chemotherapy, DMFT 17.01 (SD 9.14; $\mathrm{n}=157$ ) and 4.5 (SD 2.88; $\mathrm{n}=132$ ), respectively.

In regards to periodontal disease in cancer patients, results showed that the weighted prevalence of severe gingivitis from three cohort studies was $20.3 \%$ (SE 0.49 , 95\%CI 0-41.4). The Gingival Index (GI) for patients who were post-chemotherapy was 1.02 (SD 0.15; $\mathrm{n}=162$ ). The overall Plaque Index (PI) for patients who were post-antineoplastic therapy was 1.38 (SD $0.25 ; \mathrm{n}=189$ ) and the PI for patients who were post-chemotherapy was 1.46 (SD 0.23; $\mathrm{n}=162$ )

\section{Systemic infections from oral sources}

Febrile episodes associated with odontogenic infections have been reported in two studies that were conducted on patients undergoing intensive chemotherapy regimens. In one of the studies, the reported incidence of febrile episode originating from a dental problem was $4 \%,(n=48)$ and this finding represented a relative incidence rate of $10 \%$ for acute conversion of previously diagnosed severe chronic dental disease. ${ }^{6}$ In the second study, no source of infection other than an oral one was found in $42 \%$ of the recorded febrile episodes. ${ }^{7}$ This study also reported that patients with febrile episodes had more severe dental infection (57.6\%) than those without (23.3\%).

\section{Strengths and weaknesses of this systematic review}

While evidence of toxicity on oral mucosal lining and on salivary glands as direct consequences in chemotherapy and/or radiotherapy of head and neck cancer has been shown in well-designed studies, $^{8-10}$ identification, appraisal and synthesis of the literature on dental and periodontal complication in cancer patients had not been accomplished since 1989. Due to the significant advances in cancer therapies in the past decades (ie intensity of chemotherapy regimens, intensity modulated radiation therapy, use of radioprotective agents, etc) it is expected that new evidence on trends in prevalence, nature and severity of these oral complications has been accrued. Thus, the relevance and usefulness of this review is imperative for dental clinicians in treating these patients.

As for the review methodology, this review had a number of limitations. A key source of potential bias in a systematic review is bias due to limitations in the original studies contained within it. Regarding study selection for this review, no inclusion criteria were stated. Authors mentioned having considered scientific quality methods of assessment to determine the weighted prevalence of caries and dental infection. However, quality assessment is not clearly documented in the article. In addition, the search was restricted to English language publications and given the worldwide oral complications in cancer patients, this restriction may have introduced bias.

In relation to the clinical question about prevalence of caries and of periodontal disease, observational studies were included in the review. Almost half of them being were case control and cross sectional studies (22 of the 46). Despite the methodological quality of included studies, the authors did not mention how they treated possible bias inherent to study designs (loss to follow up, selection bias and misclassification) or potential confounding variables. Although treatment modality is one of the most relevant variables to be considered in prevalence calculation for cancer survivors, it is worth noting that in seven studies included ( $\mathrm{n}=1812 \mathrm{pts}$ ) there was no breakdown or a vague description of administered cancer therapy. In spite of the intuitive appeal of overall weighted prevalence, its dependence on prevalence from studies where the study population diverges considerably may particularly mislead the real prevalence in the cancer population setting. ${ }^{10-12}$ Unfortunately, a comprehensive list of included studies to calculate prevalence was not provided in this review. It is important to remark that although the authors reported weighted prevalence as the main outcome, methods to address and calculate weighting were not stated in the article. Moreover, it was not clear what is the total number of patients who were included for prevalence calculation. Besides clinical differences between the groups mentioned by the authors when obtaining the overall weighted prevalence of caries, the wide range of its $95 \% \mathrm{CI}$ in the three groups may suggest a small sample size in each group, or other clinical differences such as age, dif- 
ferent chemotherapeutic drugs or other medications, etc between the groups of patients included from different studies. Therefore, it is suggested that any conclusion about these findings should be drawn with caution.

Concerning the prevalence of severe gingivitis, although it was obtained only in patients undergoing chemotherapy for acute leukaemia treatment, two of them included a paediatric population, ${ }^{13,14}$ and only one study included adult patients. Within the study which included adults, $50 \%$ of patients received plaque control using $0.1 \%$ chlorhexidine solution and in the other $50 \%$ of patients this measure was preceded by mechanical removal of plaque and calculus on day one. ${ }^{15}$ This may suggest that the study population used to obtain gingivitis severity could not have been at the same baseline risk. It is worth noting that the three studies included to obtain Gingival Index were conducted to evaluate long-term effects of chemotherapy in paediatric groups. ${ }^{16-19}$ Considering the potential aggressiveness of severe gingivitis in patients who undergo high dose chemotherapy, these GI values may not be representative for this patient cancer group. The authors pointed out that attempts to describe periodontal health and periodontal disease beyond PI and GI indices in cancer patients were difficult in this review. Reasons stated were: the variety of outcome variables reported; the ambiguity in the categorisation and definition of periodontal status; and the scarcity of more representative measurements of periodontal status reported in the articles.

The authors concluded that patients who were post-radiotherapy had the highest DMFT compared to those who were post-chemotherapy and healthy controls. Even though no statistical test to analyse this difference between groups was mentioned, clinical differences between groups can explain this result. While radiation damage is anatomically site-specific, chemotherapy often can cause both site-specific and non-oral toxicity. Consequently, it is expected to see differences in the prevalence of caries and periodontal disease among these two distinct groups of patients. Because of direct radiation toxicity on the dental apparatus and because of the xerostomia resulting from degenerative radio effects on salivary gland parenchyma, a higher prevalence of caries is to be expected in patients who undergo radiotherapy. As the result of myelosupression and loss of protective salivary constituents, patients who undergo high dose chemotherapy regimens are expected to have a higher prevalence of periodontal disease. Therefore, prevalence or incidence studies disaggregated by these distinct groups of patients would be required to assess the real overall prevalence of dental and periodontal disease.

Despite the reported low prevalence of systemic infection associated with a periodontogenic or odontogenic source, there is evidence that these infections may become potentially life-threatening in myelosuppressed cancer patients. Based on this theoretical reasoning and indirect evidence, it seems reasonable to suggest that all acute and potential sources of oral infections should be correctly eradicated. Due to the distinct consequences of the presence of dental infection between patients who undergo intensive chemotherapy versus patients undergoing radiotherapy, the results of this evidence cannot be extrapolated to patients who receive ionising radiation for cancer treatment. Obviously, large prospective studies would be required to definitively address proper pre-cancer therapy clearance protocols in this patient setting.
In conclusion, this review emphasises the current scarcity of well-designed studies to evaluate the extent and severity of dental and periodontal diseases associated with complications during cancer therapy. The authors underscored the lack of specification and standardisation, and wide ranges of time periods of index values data collection.

\section{Implications for Dental Practitioners}

Oral complications from radiation to the head and neck or chemotherapy for any malignancy can compromise patients' health and affect their ability to complete planned cancer treatment. On occasions, the complications can be so debilitating that they may postpone scheduled treatments, or discontinue treatment entirely. Oral complications can also lead to serious systemic infections. Medically necessary oral care before, during and after cancer treatment can prevent or reduce the incidence and severity of oral complications, enhancing both patient survival and quality of life.

As cancer treatment is increasingly effective, an increasing number of patients receive cancer chemotherapy on an in-patient basis or outpatient basis. By identifying patients at risk of oral complications, dental practitioners are able to start preventive measures before cancer therapy begins, reducing the occurrence of problems brought about by different treatment modalities. One of the most important risk factors leading to problems is dental or periodontal disease that already exists, and poor oral care during cancer therapy. A thorough oral evaluation by a knowledgeable dentist before cancer treatment begins is important to the success of the regimen. Special considerations for haematopoietic stem cell transplant patients should be taken into account. The intensive conditioning regimens of transplantation can result in pronounced immunosuppression increasing a patient's risk of serious oral complications. Although complications begin to resolve when haematological status improves, immunosuppression may last for up to a year after the transplant, along with the risk of infections. Therefore, careful attention to oral care in the immediate and long-term post-transplant period is important to patients' overall health.

Finally, the developments in oncology demands continuous adaptation of oral care regimens and emphasises the need for supportive care in the cancer multidisciplinary team, with close cooperation between the different disciplines involved in providing oral supportive care in cancer patients. It is recommended that the needs of patients can be best met by integrated dental and medical programs in oncology. Consequently, an accurate knowledge of the burden of illness of oral complications by identifying highrisk populations, assessing of oral status and stabilisation of oral disease prior to cancer therapy is imperative for management of the numerous oral complications of cancer therapy. An advance in understanding the frequency of dental and periodontal complications to minimise risk for oral and associated systemic complications is essential for dental practitioners.

Marisol Michelet
Oral Medicine Department, FUNDALEU-Foundation to Fight
against Leukemia, Hospitalization and Clinical Research Center
Buenos Aires- Argentina

Marisol Michelet Buenos Aires- Argentina 
1. Lalla RV, Brennan MT, Schubert MM. Oral complications of cancer therapy. In Yagiela JA, Dowd FJ, Johnson BS, et al., eds. Pharmacology and Therapeutics for Dentistry. 6th ed. pp 782-798. St. Louis, Mo: Mosby Elsevier 2011.

2. Schubert MM, Peterson DE, Lloid ME. Oral complications. In Thomas ED, Blume KG, Forman SJ, eds. Hematopoietic Cell Transplantation. 2nd ed. pp 751-763. Malden, Mass: Blackwell Science Inc 1999.

3. Epstein JB, Chow AW. Oral complications associated with immunosuppression and cancer therapies. Infect Dis Clin North Am 1999; 13: 901-923.

4. Seikaly H, Jha N, McGaw T, Coulter L, Liu R, Oldring D. Submandibular gland transfer: a new method of preventing radiation-induced xerostomia. Laryngoscope 2001; 111: 347-352.

5. National Institutes of Health. Oral complications of cancer therapies: diagnosis, prevention, and treatment. National Institutes of Health Consensus Development Conference Statement, 17-19 April 1989, Vol. 7, pp 1-11.

6. Toljanic JA, Bedard JF, Larson RA, Fox JP. A prospective pilot study to evaluate a new dental assessment and treatment paradigm for patients scheduled to undergo intensive chemotherapy for cancer. Cancer 1999; 85: 1843-1848.

7. Laine PO, Lindqvist JC, Pyrhonen SO, Strand-Pettinen IM, Teerenhovi LM, Meurman $\mathrm{H}$. Oral infection as a reason for febrile episodes in lymphoma patients receiving cytostatic drugs. Eur / Cancer B Oral Oncol 1992; 28B: 103-107.

8. Keefe DM, Schubert MM, Elting LS, et al. Updated clinical practice guidelines for the prevention and treatment of mucositis. Cancer 2007; 109: 820-831.

9. Clarkson JE, Worthington HV, Eden OB. Interventions for treating oral mucositis for patients with cancer receiving treatment. Cochrane Database Syst Rev. 2007; 18;(2): CD001973.

10. Sanderson S, Tatt ID, Higgins JP. Tools for assessing quality and susceptibility to bias in observational studies in epidemiology: a systematic review and annotated bibliography· Int J Epidemiol 2007; 36: 666-676. Epub 2007 Apr 30.
11. Silva LC, Orduñez P, Paz Rodriguez MP, Robles S. A tool for assessing the usefulness of prevalence studies done for surveillance purposes: the example of hypertension. Rev Panam Salud Publica. 2001; 10: 226-231.

12. Shea B, Grimshaw JM, Wells GA, et al. Development of AMSTAR: a measurement tool to assess the methodological quality of systematic reviews. BMC Med Res Methodol 2007; 7: 10.

13. Jensen SB, Pedersen AM, Vissink A, et al. A systematic review of salivary gland hypofunction and xerostomia induced by cancer therapies: prevalence, severity and impact on quality of life. Support Care Cancer 2010; 18: 1039-1060.

14. Fayle SA, Curzon ME. Oral complications in pediatric oncology patients. Pediatr Dent 1991; 13: 289-295.

15. Baliga AM, Brave VR, Vyas HA. Oral mucosal lesions in patients with acute leukemias and related disorders due to cytotoxic therapy. J Indian Soc Pedod Prev Dent 1995, 13: 25-29.

16. Bergmann OJ, Ellegaard B, Dahl M, Ellegaard J. Gingival status during chemical plaque control with or without prior mechanical plaque removal in patients with acute myeloid leukaemia. / Clin Periodontol 1992; 19: 169-173.

17. Avsar A, Elli M, Darka O, Pinarli G. Long-term effects of chemotherapy on caries formation, dental development, and salivary factors in childhood cancer survivors. Oral Surg Oral Med Oral Pathol Oral Radiol Endod 2007; 104: 781-789.

18. Oguz A, Cetiner S, Karadeniz C, Alpaslan G, Alpaslan C, Pinarli G. Long-term effects of chemotherapy on orodental structures in children with non-Hodgkin's lymphoma. Eur / Oral Sci 2004; 112: 8-11.

19. Alpaslan G, Alpaslan C, Gogen H, Oguz A, Cetiner S, Karadeniz C. Disturbances in oral and dental structures in patients with pediatric lymphoma after chemotherapy: a preliminary report. Oral Surg Oral Med Oral Pathol Oral Radiol Endod. 1999; 87: 317-321.

Evidence-Based Dentistry (2012) 13, 70-73. doi:10.1038/sj.ebd.6400870 